\title{
On-chip Brownian relaxation measurements of magnetic nanobeads in the time domain
}

Østerberg, Frederik Westergaard; Rizzi, Giovanni; Hansen, Mikkel Fougt

Published in:

Journal of Applied Physics

Link to article, DOI:

$10.1063 / 1.4811462$

Publication date:

2013

Document Version

Publisher's PDF, also known as Version of record

Link back to DTU Orbit

Citation (APA):

Østerberg, F. W., Rizzi, G., \& Hansen, M. F. (2013). On-chip Brownian relaxation measurements of magnetic nanobeads in the time domain. Journal of Applied Physics, 113(23), 234508. https://doi.org/10.1063/1.4811462

\section{General rights}

Copyright and moral rights for the publications made accessible in the public portal are retained by the authors and/or other copyright owners and it is a condition of accessing publications that users recognise and abide by the legal requirements associated with these rights.

- Users may download and print one copy of any publication from the public portal for the purpose of private study or research.

- You may not further distribute the material or use it for any profit-making activity or commercial gain

- You may freely distribute the URL identifying the publication in the public portal 


\section{AIP Appilied Physics}

On-chip Brownian relaxation measurements of magnetic nanobeads in the time domain

Frederik Westergaard Østerberg, Giovanni Rizzi, and Mikkel Fougt Hansen

Citation: J. Appl. Phys. 113, 234508 (2013); doi: 10.1063/1.4811462

View online: http://dx.doi.org/10.1063/1.4811462

View Table of Contents: http://jap.aip.org/resource/1/JAPIAU/v113/i23

Published by the AIP Publishing LLC.

\section{Additional information on J. Appl. Phys.}

Journal Homepage: http://jap.aip.org/

Journal Information: http://jap.aip.org/about/about_the_journal

Top downloads: http://jap.aip.org/features/most_downloaded

Information for Authors: http://jap.aip.org/authors

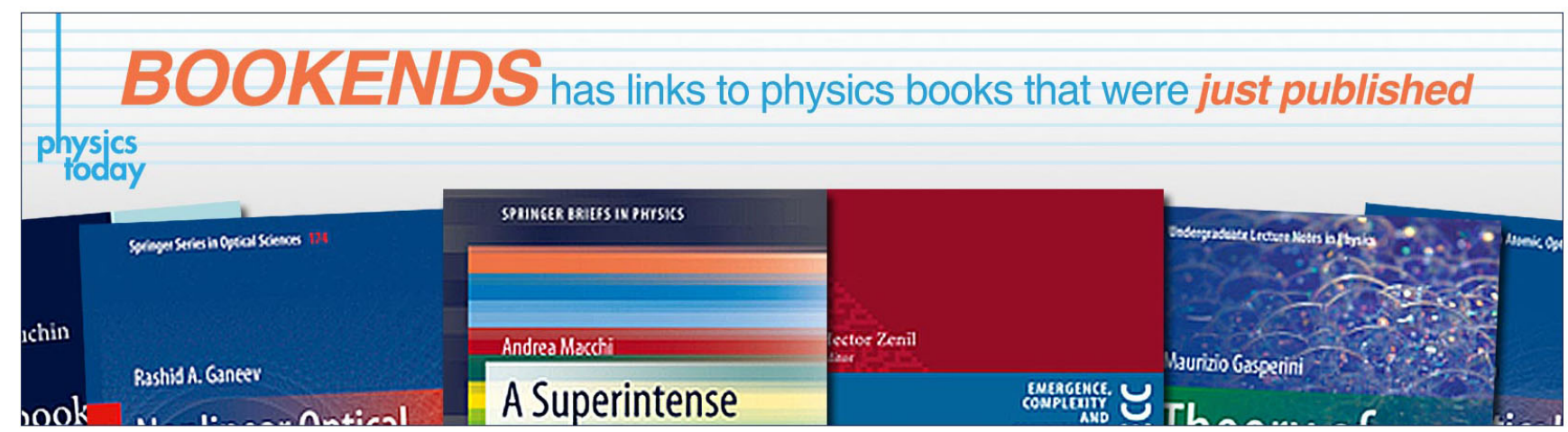




\title{
On-chip Brownian relaxation measurements of magnetic nanobeads in the time domain
}

\author{
Frederik Westergaard Østerberg, ${ }^{\text {a) }}$ Giovanni Rizzi, and Mikkel Fougt Hansen ${ }^{\text {) }}$ \\ Department of Micro- and Nanotechnology, Technical University of Denmark, DTU Nanotech, Building 345 \\ East, DK-2800 Kongens Lyngby, Denmark
}

(Received 27 March 2013; accepted 3 June 2013; published online 19 June 2013)

\begin{abstract}
We present and demonstrate a new method for on-chip Brownian relaxation measurements on magnetic nanobeads in the time domain using magnetoresistive sensors. The beads are being magnetized by the sensor self-field arising from the bias current passed through the sensors and thus no external magnetic fields are needed. First, the method is demonstrated on Brownian relaxation measurements of beads with nominal sizes of 40,80,130, and $250 \mathrm{~nm}$. The results are found to compare well to those obtained by an already established measurement technique in the frequency domain. Next, we demonstrate the time and frequency domain methods on Brownian relaxation detection of clustering of streptavidin coated magnetic beads in the presence of different concentrations of biotin-conjugated bovine serum albumin and obtain comparable results. In the time domain, a measurement is carried out in less than $30 \mathrm{~s}$, which is about six times faster than in the frequency domain. This substantial reduction of the measurement time allows for continuous monitoring of the bead dynamics vs. time and opens for time-resolved studies, e.g., of binding kinetics. C 2013 AIP Publishing LLC. [http://dx.doi.org/10.1063/1.4811462]
\end{abstract}

\section{INTRODUCTION}

Magnetic beads have been demonstrated as a useful component for the read-out in future biosensors. ${ }^{1-4}$ A major advantage of using magnetic beads is that most biological samples are nonmagnetic and, therefore, there is nominally no background signal from the sample. Magnetic beads are also easy to functionalize such that they bind specifically to, e.g., antibodies, proteins, or DNA.

Traditionally, biosensing with a read-out based on magnetic beads is either surface-based or volume-based. In surface-based sensing, both the sensor surface and the beads are functionalized such that the target analyte will be sandwiched between the beads and the surface and result in beads being bound to the sensor surface. ${ }^{2,5,6}$ In volume-based sensing, only the beads are functionalized and the presence of the target analyte is detected as a change of the hydrodynamic size of the magnetic beads either by adding to the size of the beads or by inducing bead agglutination. The use of Brownian relaxation measurements for volume-based biosensing was first proposed by Connolly and St Pierre. ${ }^{7}$

Brownian relaxation of magnetic beads is often measured in the frequency domain by $\mathrm{AC}$ susceptibility measurements with either inductive methods, ${ }^{8}$ fluxgates, ${ }^{9}$ SQUID magnetometers, ${ }^{10}$ or magnetoresistive sensors. ${ }^{11,12}$ However, Brownian relaxation can also be measured in the time domain. ${ }^{13-15}$ The advantage of time domain measurements is that they can be performed much faster and a single measurement may take less than a second, whereas frequency domain measurements require a sequence of measurements at different frequencies typically resulting in measurement times on the order of minutes or longer. Thus, time domain

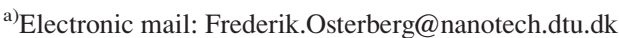

${ }^{b)}$ Electronic mail: Mikkel.Hansen@nanotech.dtu.dk
}

measurements result in a much shorter analysis time and therefore also enable time-resolved studies. Disadvantages of time domain measurements compared to frequency domain measurements are that more noise are picked up in a DC measurement compared to the lock-in technique used in the frequency domain, and the time resolution of the data acquisition will set a limit for how fast changes can be detected.

Recently, we have demonstrated the possibility of performing Brownian relaxation measurements in the frequency domain on so called planar Hall effect bridge sensors without the need for any external magnets. ${ }^{16,17}$ Here, we present and demonstrate a new approach that enables the use of the same sensors for Brownian relaxation measurements in the time domain and we show that this new approach substantially reduces the measuring time. We first compare measurements in the frequency and time domains on a series of four nanobead samples with diameters ranging from $40 \mathrm{~nm}$ to $250 \mathrm{~nm}$. Next, we present initial results of biodetection experiments, where the two methods are employed to detect the clustering of streptavidin beads in the presence of biotin-conjugated bovine serum albumin.

\section{THEORY}

\section{A. Brownian relaxation of magnetic beads}

We consider an ensemble of magnetic beads placed in an applied magnet field with an equilibrium magnetization $M_{0}$. For convenience, the measured magnetization $M$ is defined to be positive when it is parallel to the applied field, i.e., the magnetization $M$ equals $+M_{0}$ and $-M_{0}$ when the magnetic moments are parallel and antiparallel to the applied field, respectively. The bead magnetic moments align with the applied magnetic field either by internal flipping of the 
magnetization (Néel relaxation ${ }^{18}$ ) or by a physical rotation $\left(\right.$ Brownian relaxation ${ }^{19}$ ). For the beads used in this study, we assume that the Néel relaxation time is much longer than the Brownian relaxation time, which therefore dominates the relaxation dynamics of the beads. ${ }^{17}$ The Brownian relaxation of magnetic beads can be measured in both the time and the frequency domains. The theory for both domains is described below.

\section{Time domain}

The Brownian relaxation of magnetic beads suspended in a liquid is characterized by the characteristic Brownian relaxation time

$$
\tau_{\mathrm{B}}=\frac{3 \eta V_{\mathrm{h}}}{k_{\mathrm{B}} T},
$$

where $\eta$ is the viscosity of the liquid, $V_{\mathrm{h}}$ is the hydrodynamic volume of the bead, and $k_{\mathrm{B}} T$ is the thermal energy.

In this study, the measurements in the time domain are performed by passing a square wave current $I(t)$ with an amplitude of $I_{0}$ and period $T_{I}$ though the sensor. The current through the sensor generates a magnetic self-field $(H)$ around the sensor, which is proportional to the current, such that the sign of the magnetic field changes with the sign of the current. The beads rotate to align their magnetic moments with the magnetic field. This means that just after flipping of the current from $+I_{0}$ to $-I_{0}$, the magnetization of the beads will be antiparallel with the sensor self-field and then relax to being parallel. This is repeated when the current changes sign from $-I_{0}$ to $+I_{0}$, where it is noted that $M$ again has an initial value of $-M_{0}$ as it is antiparallel to the magnetic field generated by the current. This is illustrated in Fig. 1.

The relaxation of the beads is described by an exponential decay with the Brownian relaxation time as the exponential time constant. As the magnetization of the beads relaxes from being antiparallel $\left(-M_{0}\right)$ to being parallel $\left(+M_{0}\right)$ with the magnetic field, we assume it to be described by

$$
M(t)=M_{0}\left[1-2 \exp \left(-t / \tau_{\mathrm{B}}\right)\right],
$$

where $t$ is the time after the sign change of the magnetic field.

\section{Frequency domain}

The traditional method of measuring Brownian relaxation with planar Hall effect sensors is to pass an alternating current through the sensor

$$
I(t)=\sqrt{2} I_{\mathrm{RMS}} \sin (2 \pi f t),
$$

where $I_{\text {RMS }}$ is the root mean square value of the current and $f$ is the frequency of the current.

Measurements in the frequency domain are characterized by the complex magnetic susceptibility $\chi=\chi^{\prime}-\mathrm{i} \chi^{\prime \prime}$ with the in-phase component $\chi^{\prime}$ and out-of-phase component $\chi^{\prime \prime}$. The Brownian relaxation is characterized by the Brownian relaxation frequency

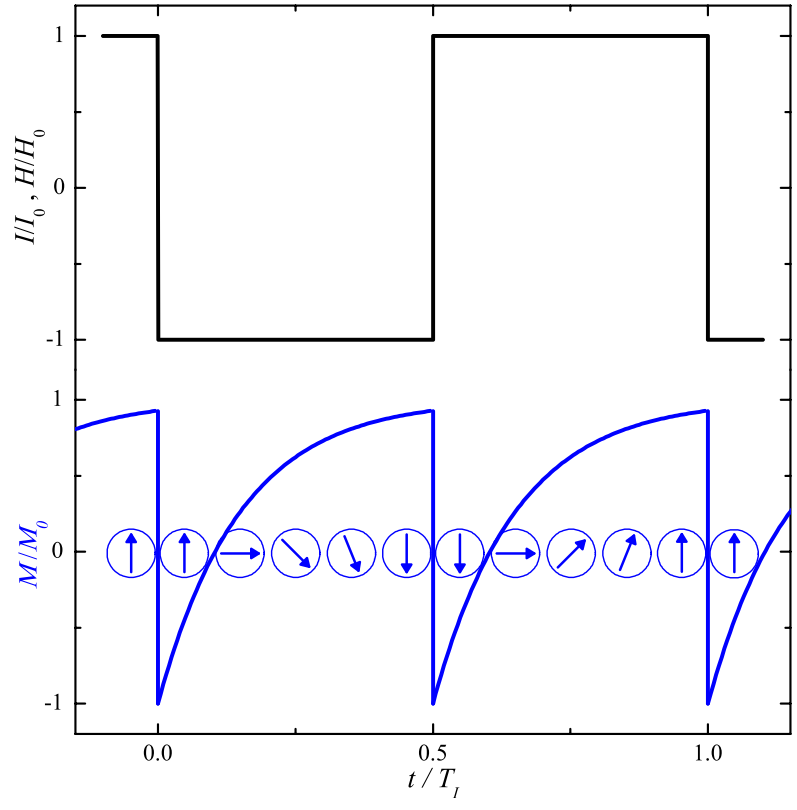

FIG. 1. Schematic of how a magnetic bead relaxes in a flipping magnetic field. The magnetic field changes direction when the current changes direction. Before flipping of the magnetic field, the magnetization of the bead will be parallel with the field. Immediately after flipping of the magnetic field, the magnetization of the bead will be anti-parallel, meaning the magnetization along the applied field is $M=-M_{0}$. The bead will then relax by rotation to become parallel with the field $\left(M=M_{0}\right)$.

$$
f_{\mathrm{B}}=\frac{1}{2 \pi \tau_{\mathrm{B}}}=\frac{k_{\mathrm{B}} T}{6 \pi \eta V_{\mathrm{h}}} .
$$

At this characteristic frequency, the phase-lag between the magnetic moment of the bead and the applied field is largest, meaning that a peak will appear in the out-of-phase magnetic susceptibility at $f=f_{\mathrm{B}}$.

According to Debye theory, ${ }^{20}$ the complex magnetic susceptibility for an ensemble of beads with a single relaxation time is given by

$$
\chi(f)=\frac{\chi_{0}-\chi_{\infty}}{1+\left(\mathrm{i} f / f_{\mathrm{B}}\right)}+\chi_{\infty}
$$

where $\chi_{0}$ and $\chi_{\infty}$ are the DC and high-frequency susceptibilities, respectively.

\section{Size distribution}

The equations for the magnetization in the time domain and the magnetic susceptibility in the frequency domain are only valid for a monodisperse ensemble of beads. Hence, the equations need to be averaged over a bead distribution. The size distribution of magnetic beads is often well described by the log-normal distribution given by

$$
f_{\mathrm{LN}}\left(D_{\mathrm{h}}\right) \mathrm{d} D_{\mathrm{h}}=\frac{1}{D_{\mathrm{h}} \sigma \sqrt{2 \pi}} \exp \left(-\frac{\left(\ln D_{\mathrm{h}}-\mu\right)^{2}}{2 \sigma^{2}}\right) \mathrm{d} D_{\mathrm{h}}
$$

where $\mu$ is the logarithm to the median hydrodynamic diameter $D_{\mathrm{hm}}, D_{\mathrm{hm}}=\exp (\mu)$, and $\sigma$ is the logarithmic standard deviation. We define the size distribution function to be volume-weighted, i.e., the volume fraction of the particles 
with hydrodynamic diameters between $D_{\mathrm{h}}$ and $D_{\mathrm{h}}+\mathrm{d} D_{\mathrm{h}}$ is $f_{\mathrm{LN}}\left(D_{\mathrm{h}} ; \mu, \sigma\right) \mathrm{d} D_{\mathrm{h}}$. The definition of the size distribution therefore takes directly into account that the signal from a single bead is proportional to the bead volume.

\section{B. Sensor signals}

The magnetic beads are magnetized by the self-field arising from the bias current passed through the sensor. Figure 2 shows the contours of the magnetic field $\mu_{0} H$ calculated using the Biot-Savart law for a total bias current of $14.1 \mathrm{~mA}$, which is the current relevant for our experimental studies. The bias current is distributed between the two sensor branches that each has the width $w=20 \mu \mathrm{m}$. The calculations show that the maximum value of $\mu_{0} H$ found near the sensor edge is about $0.25 \mathrm{mT}$. This value is well below typical values applied in Brownian relaxation measurements in commercial set-ups.

For low magnetic fields, the sensor signal is linear and given by ${ }^{21}$

$$
V=S_{0} I H_{y}+R_{\text {off }} I
$$

where $S_{0}$ is the low-field sensitivity, $H_{y}$ is the average magnetic field acting on the sensor area in the $y$-direction, and $R_{\text {off }}$ accounts for a possible sensor offset. The value of $H_{y}$ can be written as

$$
H_{y}=\gamma_{0} I+H_{y, \text { beads }}+H_{y, \text { ext }} .
$$

Here, $\gamma_{0}$ is a constant that describes the effect of the selffield and which depends on the sensor stack and geometry; ${ }^{22}$ $H_{y, \text { beads }}$ and $H_{y \text {,ext }}$ are magnetic fields acting on the sensor in the $y$-direction from the beads and external sources, respectively. The expression for $H_{y \text {, beads }}$ will be written slightly differently for measurements in the time domain and the frequency domain. In the time domain, we write

$$
H_{y, \text { beads }}^{\text {time }}=\gamma_{1 t} M(t) I
$$

and in the frequency domain, we write

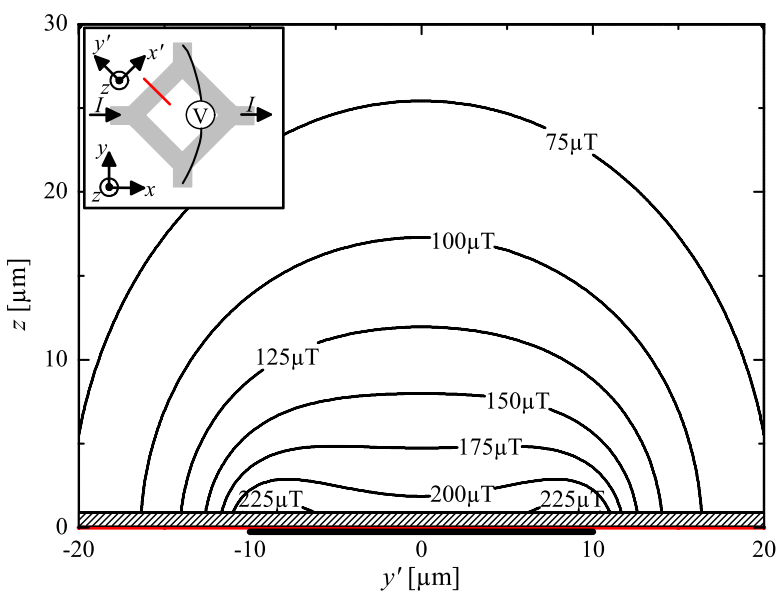

FIG. 2. Contour plot of the magnetic self-field $\left(\mu_{0} H\right)$ from the bias current through the sensor. The black line from $y^{\prime}=-10 \mu \mathrm{m}$ to $y^{\prime}=10 \mu \mathrm{m}$ represents the sensor. The inset shows a sketch of the sensor, where the red line through the upper left branch represents the cross section where the selffield is calculated.

$$
H_{y, \text { beads }}^{\text {freq }}=\gamma_{1} \chi(f) I
$$

where $\gamma_{1 t}$ and $\gamma_{1}$ are constants that depend on the sensor geometry and bead distribution. Hansen et al. ${ }^{22}$ have shown that magnetic beads magnetized by the self-field always contribute with a positive sign to the average magnetic field experienced by the sensor and that $85 \%$ of the sensor signal for a homogeneous bead dispersion arises from a volume centered on the sensor stripe with a width of about $2 w$ and height of about $1.5 w$. For the sensors in the present study, where each branch has a length $l=280 \mu \mathrm{m}$, this corresponds to a total probing volume of about $1.3 \mathrm{nl}$.

\section{Time domain}

When measurements are performed in the time domain, the current is alternating between $+I_{0}$ and $-I_{0}$ with the corresponding signals $V_{+}(t)$ and $V_{-}(t)$ given by

$$
\begin{aligned}
& V_{+}(t)=S_{0} I_{0}^{2}\left(\gamma_{0}+\gamma_{1 t} M(t)\right)+I_{0}\left(S_{0} H_{y, \text { ext }}+R_{\mathrm{off}}\right), \\
& V_{-}(t)=S_{0} I_{0}^{2}\left(\gamma_{0}+\gamma_{1 t} M(t)\right)-I_{0}\left(S_{0} H_{y, \text { ext }}+R_{\mathrm{off}}\right),
\end{aligned}
$$

where $t$ is defined as zero at the instance where the current changes sign. From this, it is seen that $R_{\text {off }}$ and $H_{y, \text { ext }}$ can be eliminated by calculating the average of the two measurements and that $\gamma_{0}+\gamma_{1 t} M(t)$ can be eliminated by calculating the difference of the two measurements

$$
\begin{aligned}
& V_{\text {ave }}(t)=\frac{1}{2}\left[V_{+}(t)+V_{-}(t)\right]=S_{0} I_{0}^{2}\left(\gamma_{0}+\gamma_{1 t} M(t)\right), \\
& V_{\text {diff }}(t)=\frac{1}{2}\left[V_{+}(t)-V_{-}(t)\right]=I_{0}\left(S_{0} H_{y, \text { ext }}+R_{\text {off }}\right) .
\end{aligned}
$$

The offset due to $\gamma_{0}$ in $V_{\text {ave }}$ can be corrected for by subtracting a measurement performed without beads. After this correction, $V_{\text {ave }}(t)$ is proportional to the time-dependent magnetization. It is seen that $V_{\text {diff }}(t)$ is linearly dependent on $H_{y, \text { ext }}$.

The function that is fitted to the measurements is the theoretical signal integrated over the volume-weighted log-normal distribution of hydrodynamic diameters

$$
V_{\mathrm{fit}, t}=-V_{0 t}\left(1-2 \int_{0}^{\infty} \exp \left[-t / \tau_{\mathrm{B}}\left(D_{\mathrm{h}}\right)\right] f_{\mathrm{LN}}\left(D_{\mathrm{h}}\right) \mathrm{d} D_{\mathrm{h}}\right)+V_{\text {off }}
$$

where $V_{0 t} \equiv-S_{0} I_{0}^{2} \gamma_{1 t} M_{0}$ is the amplitude of the decay and $V_{\text {off }}$ is correcting for possible offsets. With these definitions, $V_{0 t}$ is positive (as $S_{0}<0$ ) and $V_{\text {fit }, t} / V_{0 t}$ equals +1 for $t=0$ and -1 for $t \rightarrow \infty$. The free fitting parameters are $V_{0 t}, V_{\text {off }}, D_{\mathrm{hm}}$, and $\sigma$, where the two last parameters account for the distribution of hydrodynamic diameters.

\section{Frequency domain}

For measurement in the frequency domain using lock-in detection, we have previously shown ${ }^{16}$ that the complex magnetic susceptibility can be obtained from the complex 
second harmonic sensor signal $V_{2}=V_{2}^{\prime}+\mathrm{i} V_{2}^{\prime \prime}$ with in-phase and out-of-phase components

$$
\begin{gathered}
V_{2}^{\prime}=-2^{-2} I_{\mathrm{RMS}}^{2} S_{0} \gamma_{1} \chi^{\prime \prime}, \\
V_{2}^{\prime \prime}=-2^{-2} I_{\mathrm{RMS}}^{2} S_{0}\left(\gamma_{0}+\gamma_{1} \chi^{\prime}\right) .
\end{gathered}
$$

Thus, the in-phase second harmonic sensor signal is proportional to the out-of-phase magnetic bead susceptibility and the out-of-phase second harmonic sensor signal depends linearly on the in-phase magnetic bead susceptibility. Again, the offset due to $\gamma_{0}$ can be corrected for by subtracting a measurement without beads.

The function used for fitting to the measurements in the frequency is again the sensor signal averaged over the volume-weighted lognormal distribution of hydrodynamic diameters

$$
\begin{gathered}
V_{\mathrm{fit}, f}=V_{2}^{\prime}+\mathrm{i} V_{2}^{\prime \prime}, \\
=\mathrm{i} \int_{0}^{\infty} \frac{V_{0}-V_{\infty}}{1+\left(\mathrm{i} f / f_{\mathrm{B}}\left(D_{\mathrm{h}}\right)\right)} f_{\mathrm{LN}}\left(D_{\mathrm{h}}\right) \mathrm{d} D_{\mathrm{h}}+\mathrm{i} V_{\infty},
\end{gathered}
$$

with $V_{0}=-2^{-2} I_{\mathrm{RMS}}^{2} S_{0} \gamma_{1} \chi_{0}$ and $V_{\infty}=-2^{-2} I_{\mathrm{RMS}}^{2} S_{0} \gamma_{1} \chi_{\infty}$. In addition to $V_{0}$ and $V_{\infty}$, the free fitting parameters are the median hydrodynamic diameter $D_{\mathrm{hm}}$ and the logarithmic standard deviation $\sigma$.

\section{EXPERIMENTAL}

\section{A. Sensor fabrication and mounting}

The magnetic field sensor used is a so called planar Hall effect bridge sensor based on the anisotropic magnetoresistance of permalloy. ${ }^{16,21}$ The sensor stack $\mathrm{Ta}(3 \mathrm{~nm}) /$ $\mathrm{Ni}_{80} \mathrm{Fe}_{20}(30 \mathrm{~nm}) / \mathrm{Mn}_{80} \mathrm{Ir}_{20}(20 \mathrm{~nm}) / \mathrm{Ta}(3 \mathrm{~nm})$ was deposited in a field of $20 \mathrm{mT}$ applied along the positive $x$-direction to define an easy magnetization direction. The stack was patterned in a Wheatstone bridge geometry consisting of four segments each with a length of $l=280 \mu \mathrm{m}$ and a width of $w=20 \mu \mathrm{m}$. For a more detailed description of the sensor fabrication and design, see Ref. 16. The low-field sensitivity of the sensor was measured to $S_{0} / \mu_{0}=-591 \mathrm{~V} /(\mathrm{T} \mathrm{A})$ and the resistance along the current direction was measured to $161.7 \Omega$.

In order to allow for electrical contact to the sensor, a click-on fluidic system ${ }^{12,16}$ was used, which also defined the fluidic channel with dimensions length $\times$ width $\times$ height $=5 \mathrm{~mm} \times 1 \mathrm{~mm} \times 0.1 \mathrm{~mm}$. The temperature of the sensor mount in good thermal contact to the sensor was kept constant at $(25.00 \pm 0.01)^{\circ} \mathrm{C}$ by a Peltier element. The sensor was neither electrically nor magnetically shielded.

\section{B. Measurement equipment}

The current through the sensor was supplied by a 6221 AC and DC Current Source (Keithley Instruments, USA). For time domain measurements, it delivered a square wave with a current amplitude of $I_{0}=14.1 \mathrm{~mA}$ at a frequency of $8 \mathrm{~Hz}$, which allowed $T_{I} / 2=62.5 \mathrm{~ms}$ between each flipping of the current. For measurements in the frequency domain, a sine wave with a current amplitude of $20 \mathrm{~mA}\left(I_{\mathrm{RMS}}=14.1 \mathrm{~mA}\right)$ was used with a frequency varying in 29 logarithmically equidistant steps from $43.69 \mathrm{kHz}$ to $1.88 \mathrm{~Hz}$. For both domains, a trigger signal was sent from the power supply, such that the measurements in the time domain were started exactly at the point when the current was flipped and, in the frequency domain, to get accurate information on the phase.

Measurements in the time domain were recorded using a NI USB-6281 data acquisition card (National Instruments, USA) with 18 bits of resolution on a range $\pm 0.1 \mathrm{~V}$. This corresponds to a voltage resolution of $0.8 \mu \mathrm{V}$. The sample rate was set to 600000 samples per second, and in addition, a low pass filter of $3 \mathrm{kHz}$ was applied to reduce the impact of highfrequency noise. To improve the signal-to-noise ratio, the signal was averaged over 192 periods. Thus, the time used for each measurement in the time domain was $24 \mathrm{~s}$. For the data analysis, the measurement data were combined and averaged in 500 "bins" of widths increasing logarithmically with time.

Measurements of the second harmonic sensor signal in the frequency domain were recorded using a SR830 lock-in amplifier (Stanford Research Systems, USA). A frequency sweep consisting of measurements at 30 frequencies took 2 min and $21 \mathrm{~s}$.

The data acquisition card and the lock-in amplifier were connected in parallel such that measurements with both instruments were performed immediately after one another. The measurement time in the time domain of $24 \mathrm{~s}$ is about a factor of six smaller than the measurement time in the frequency domain of $2 \mathrm{~min}$ and $21 \mathrm{~s}$.

\section{Procedure}

\section{Bead sizes}

The Brownian relaxation has been measured in both the time and frequency domains for four different bead types with nominal diameters of $40 \mathrm{~nm}, 80 \mathrm{~nm}, 130 \mathrm{~nm}$, and $250 \mathrm{~nm}$. The $40 \mathrm{~nm}$ beads were SHP Iron oxide nanoparticles with carboxylic acid groups (Ocean Nanotech, USA), the $80 \mathrm{~nm}$ beads were BNF-starch with a plain surface (Micromod, Germany), and the $130 \mathrm{~nm}$ and $250 \mathrm{~nm}$ were Nanomag-D also with plain surfaces (Micromod, Germany).

All bead suspensions were diluted to a bead concentration of $1 \mathrm{mg} / \mathrm{ml}$ with milliQ water for the $40 \mathrm{~nm}$ beads and a phosphate buffered saline (PBS) solution for the remaining three bead types. Prior to the first experiment, the fluidic channel was rinsed and filled with milliQ water. An experiment was carried out by first injecting $20 \mu \mathrm{l}$ of the sample into the fluidic channel at a flow rate of $13.3 \mu \mathrm{l} / \mathrm{min}$ for $1.5 \mathrm{~min}$. Then, the beads were left in the channel for $10 \mathrm{~min}$ to allow for a steady state and the measurements were recorded. Finally, the beads were washed out with PBS at a flow rate of $300 \mu \mathrm{l} / \mathrm{min}$ for $1-2 \mathrm{~min}$. Reference measurements with no beads were performed between measurements on the four samples. These measurements were used to correct for $\gamma_{0}$ and an instrumental phase-shift.

\section{Bead clustering}

Measurements of bead clustering were carried out by mixing $15 \mu \mathrm{l}$ of PBS containing varying concentrations of 
biotin-conjugated bovine serum albumin (bBSA) (A8549, Sigma-Aldrich, USA) with $15 \mu \mathrm{l}$ of a $2 \mathrm{mg} / \mathrm{ml}$ suspension of streptavidin coated $80 \mathrm{~nm}$ BNF-starch beads (Micromod, Germany). Each bBSA has 8-16 biotin molecules available for binding to a streptavidin molecule. Since each bBSA molecule is able to bind more than two streptavidin molecules, adding bBSA to streptavidin coated beads will allow for the formation of dimers or clusters. All concentrations given below are the bead and bBSA concentrations after mixing. Immediately after mixing, the suspension was placed in a magnetic field of $45 \mathrm{mT}$ for $10 \mathrm{~min}$ to enhance formation of bead clusters after which it was injected into the fluidic system at a flow rate of $13.3 \mu \mathrm{l} / \mathrm{min}$ for $1.5 \mathrm{~min}$. The sample was left stagnant in the fluidic system for $20 \mathrm{~min}$, allowing time for five repeated measurements in the time and frequency domains. The first measurement in the frequency domain was performed during the sample injection and was not used for the analysis. When a series of measurements was completed, the bead suspension was washed out with PBS at a flow rate of $300 \mu \mathrm{l} / \mathrm{min}$ for 1-2 min and a reference measurement (used to correct for $\gamma_{0}$ ) was performed before a new sample was injected.

Five different samples were investigated with final bBSA concentrations of $0 \mathrm{nM}, 0 \mathrm{nM}, 2.5 \mathrm{nM}, 5 \mathrm{nM}$, and $10 \mathrm{nM}$, respectively.

\section{RESULTS}

\section{A. Time domain signal vs. bias current}

Figure 3 shows equilibrium values of $V_{\text {ave }}$ and $V_{\text {diff }}$ obtained from time domain measurements vs. current amplitude $I_{0}$. The values of $V_{\text {ave }}$ in Fig. 3(a) have been corrected for $\gamma_{0}$ using the average of the two measurements without beads recorded before and after beads were on the sensor. The figure shows corrected data obtained with and without a suspension of $80 \mathrm{~nm}$ magnetic beads in the fluidic channel. In the absence of the magnetic beads, the data are scattered around zero, and in the presence of the bead suspension, the data are proportional to $I_{0}^{2}$ in accordance with Eq. (13). This shows that the data correction procedure works as intended and that the measurements are stable over time. Fig. 3(b) shows the corresponding values of $V_{\text {diff. }}$. These are found to depend linearly on $I_{0}$ in accordance with Eq. (14) with a slope that has no systematic dependence on the measurement conditions. The observed slope change can be due to a variation of the background external field and/or a change of the sensor offset between the measurements. Assuming the former, it corresponds to field changes on the order of $0.5 \mu \mathrm{T}$ and the latter, it corresponds to sensor offset changes on the order of $0.5 \mathrm{~m} \Omega$. Given that the sensor operates with no magnetic shielding, field variations on the order of $0.5 \mu \mathrm{T}$ are quite likely over a period of hours. As long as the field and offset are stable over the duration of a period in the current cycle (125 $\mathrm{ms}$ in the present experiments), it will not affect the measurements of $V_{\text {ave }}$.

\section{Bead sizes}

Figure 4 shows the time domain Brownian relaxation measurements obtained for the four different bead sizes. The

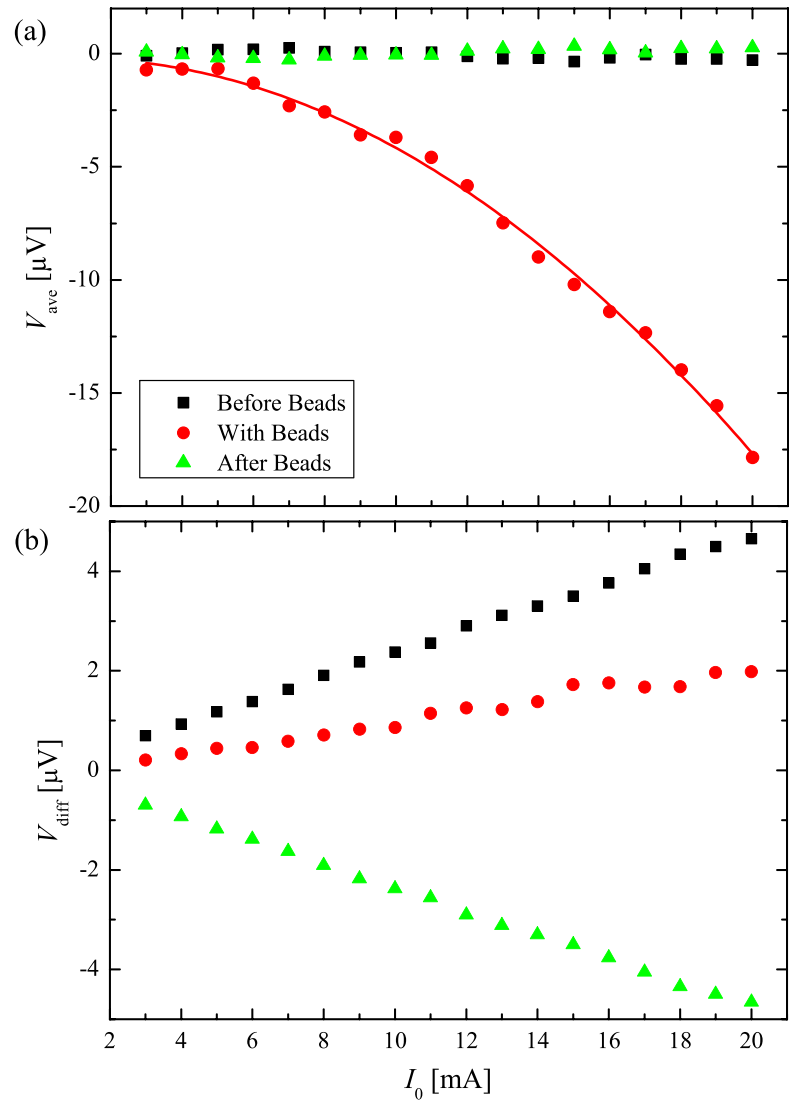

FIG. 3. Equilibrium values of $V_{\text {ave }}$ and $V_{\text {diff }}$ obtained from time domain measurements vs. current amplitude $I_{0}$. Panel (a) shows corrected equilibrium values of $V_{\text {ave }}$ vs. $I_{0}$ with and without $80 \mathrm{~nm}$ magnetic beads. The solid line is a parabolic fit to the measurements with beads. Panel (b) shows values of $V_{\text {diff }}$ vs. $I_{0}$.

measurements have been normalized with $V_{0 t}$ such that the signals for the different bead sizes can be easily compared. It is seen that the relaxation time increases with the bead size. The points measured for the $40 \mathrm{~nm}$ and $130 \mathrm{~nm}$ beads, where the absolute signal is lower, are more scattered on this normalized scale. The lines plotted through each data set are least squares curve fits of Eq. (15) to the data prior to normalization. The parameters obtained from the fits are shown in

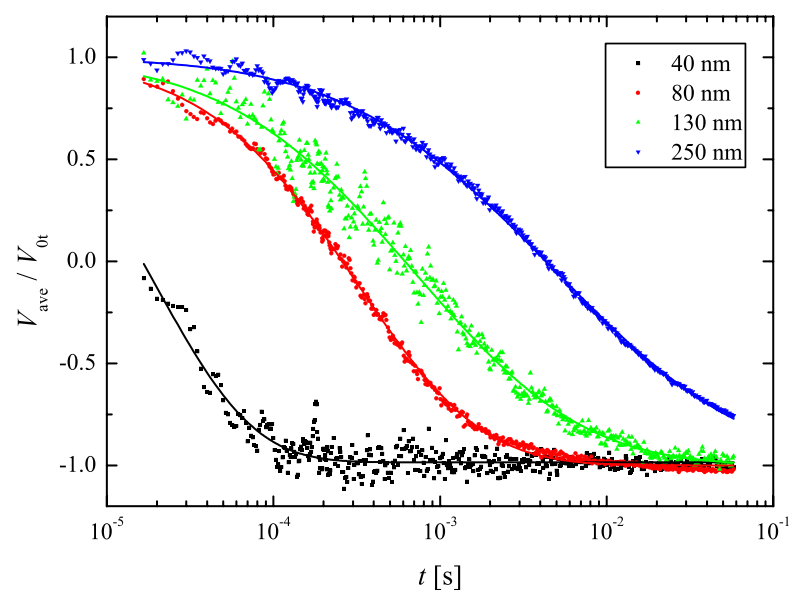

FIG. 4. Brownian relaxation measurements in the time domain. $V_{\text {ave }}$ normalized with the fitting parameter $V_{0 t}$ vs. time for four different bead sizes. The lines are curve fits of Eq. (15) to the data. The signals have all been corrected for offsets found from fitting. 
TABLE I. Parameters obtained from least squares fitting of Eq. (15) to the time domain measurements and of Eq. (19) to the frequency domain measurements for the four different bead sizes. The $40 \mathrm{~nm}$ beads are from Ocean Nanotech and suspended in MilliQ water, while the remaining three types are from Micromod and suspended in PBS. The numbers in parentheses are the errors for the $95 \%$ confidence interval obtained from the least squares curve fits.

\begin{tabular}{|c|c|c|c|c|c|c|c|c|}
\hline \multirow[b]{2}{*}{$D_{\text {nom }}[\mathrm{nm}]$} & \multicolumn{4}{|c|}{ Time domain } & \multicolumn{4}{|c|}{ Frequency domain } \\
\hline & $D_{\mathrm{hm}}[\mathrm{nm}]$ & $\sigma$ & $V_{0 t}[\mu \mathrm{V}]$ & $V_{\text {off }}[\mu \mathrm{V}]$ & $D_{\mathrm{hm}}[\mathrm{nm}]$ & $\sigma$ & $V_{0}-V_{\infty}[\mu \mathrm{V}]$ & $V_{\infty}[\mu \mathrm{V}]$ \\
\hline 40 & $41(2)$ & $0.22(5)$ & $1.8(3)$ & $-0.4(3)$ & $42.5(3)$ & $0.18(2)$ & $0.95(2)$ & $0.1(2)$ \\
\hline 80 & $108(1)$ & $0.35(1)$ & $4.81(2)$ & $0.48(2)$ & $107(2)$ & $0.32(2)$ & $3.16(5)$ & $0.4(8)$ \\
\hline 130 & $152(2)$ & $0.45(2)$ & $1.36(2)$ & $1.85(1)$ & $159(4)$ & $0.50(2)$ & $0.97(2)$ & $0.5(3)$ \\
\hline 250 & 299(4) & $0.60(1)$ & $7.68(6)$ & $8.15(5)$ & $350(7)$ & $0.64(2)$ & $5.66(7)$ & $5.5(5)$ \\
\hline
\end{tabular}

Table I. For the $40 \mathrm{~nm}$ beads from Ocean Nanotech, the median hydrodynamic diameter is found to match its nominal value within the experimental uncertainty. For the Micromod beads, the median hydrodynamic diameters are found to be significantly larger than their nominal values. The values of $\sigma$ are also found to increase with the bead size.

Figure 5 shows the Brownian relaxation measurements on the same samples in the frequency domain. As for the time domain measurements (Table I), the signal amplitude varies significantly with the bead type. The lines are least squares curve fits of Eq. (19) to the measurements. It is seen that the fits match the measured data well except for the $250 \mathrm{~nm}$ beads. The parameters obtained from the fits are listed in Table I. For beads with nominal sizes below $250 \mathrm{~nm}$, the median hydrodynamic diameters and values of $\sigma$

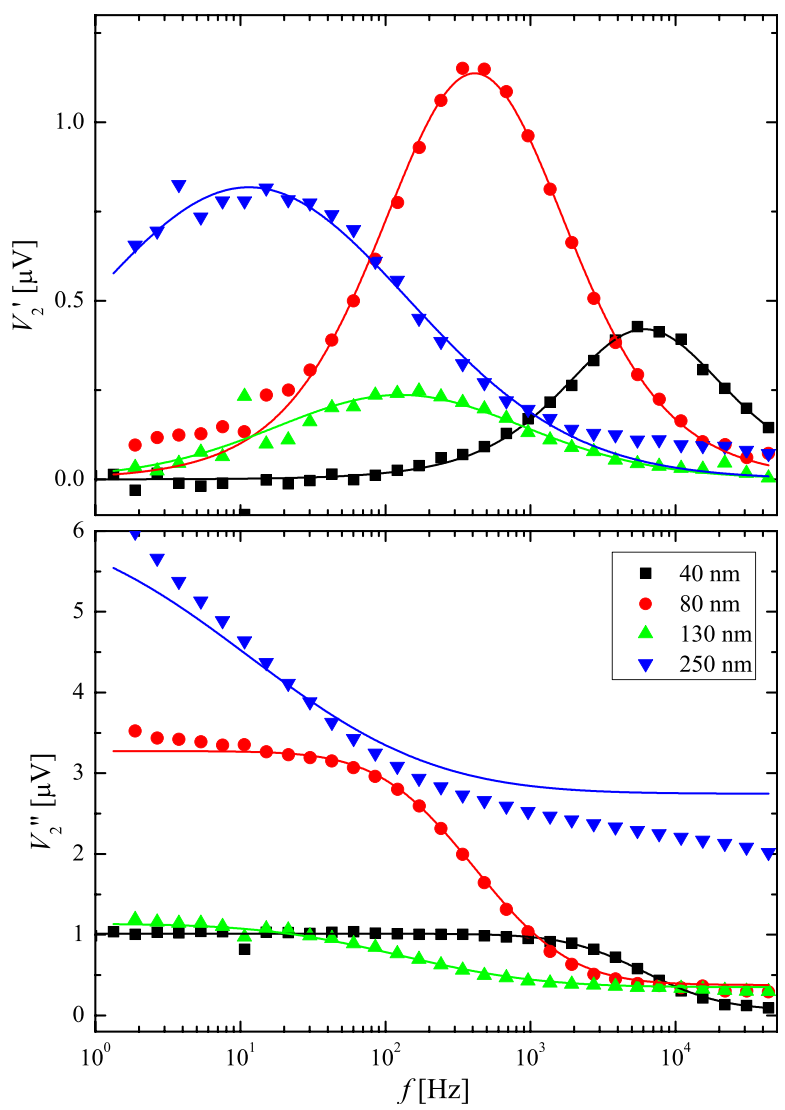

FIG. 5. Brownian relaxation measurements in the frequency domain. Second harmonic in-phase (top) and out-of-phase (bottom) signal vs. frequency for 5 different bead sizes. The sweep is performed from high to low frequencies. The lines are curve fits of Eq. (19) to the data. obtained by the two methods agree within the experimental uncertainty. However, for the $250 \mathrm{~nm}$ beads, the hydrodynamic diameter obtained from the frequency domain measurement is significantly larger than that obtained from the time domain measurement.

\section{B. Bead clustering}

Figure 6 shows time domain measurements for bead clustering with bBSA. The measurements shown are the last performed in the series of five repeated measurements and have been corrected for offsets. The lines are least squares curve fits of Eq. (15) to the data. Data from seven different measurements are shown: Two measurements with beads but without bBSA; three measurements with beads and with varying bBSA concentrations; and finally two reference measurements with neither beads nor bBSA. It is seen that the two measurements without bBSA almost coincide. When the bBSA concentration is increased, both the signal amplitude and the signal slope for $t \geq 10^{-2} \mathrm{~s}$ increase.

Figure 7 shows the corresponding measurements in the frequency domain. Again, the measurements on the two samples without bBSA are found to be very similar. For the samples with bBSA, the peak in the in-phase sensor signal (corresponding to the out-of-phase magnetic susceptibility) is found to increase in intensity and it is shifted towards lower frequencies upon increasing bBSA concentration. Least squares curve fits of Eq. (19) are plotted as solid lines.

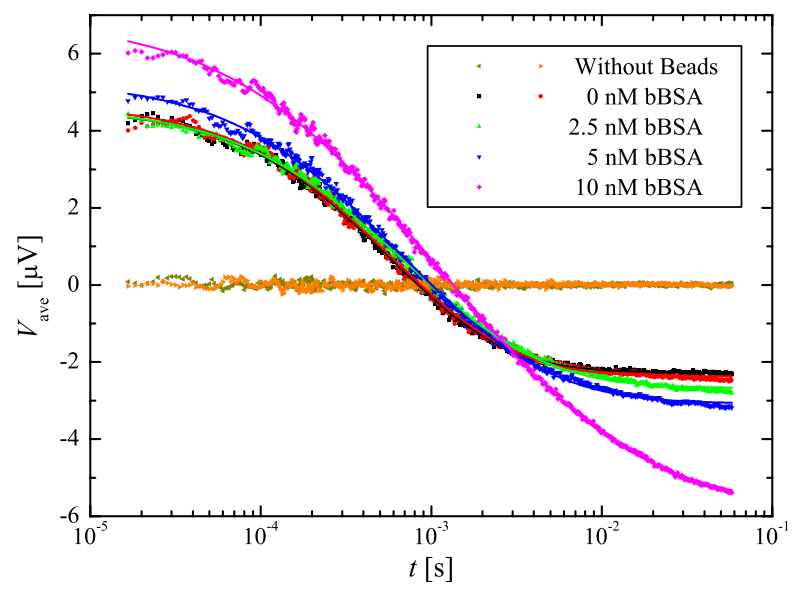

FIG. 6. Time domain measurements of clustering of streptavidin coated bead by binding to bBSA. $V_{\text {ave }}$ is plotted vs. time. The lines are curve fits of Eq. (15) to the data. 


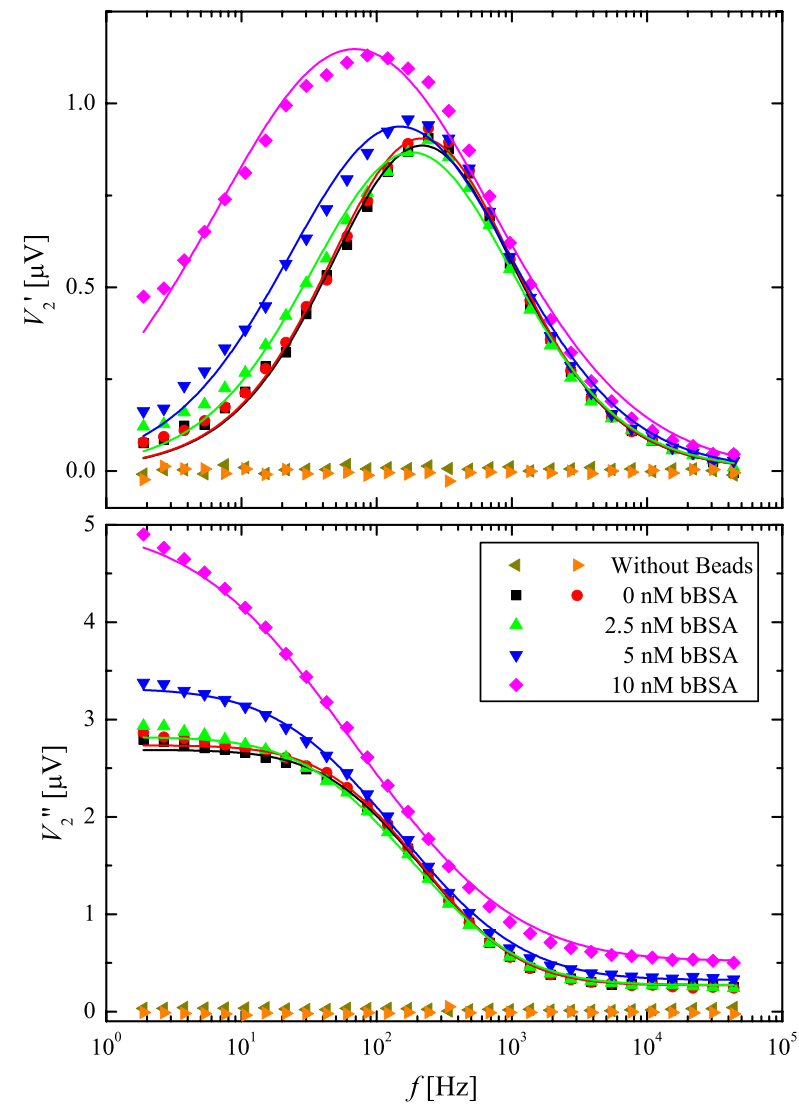

FIG. 7. Frequency domain measurements of clustering of streptavidin coated beads by bBSA. The in-phase (top) and out-of-phase (bottom) second harmonic sensor signals are plotted vs. bias current frequency. The lines are curve fits of Eq. (19) to the data.

The median hydrodynamic diameters $D_{\mathrm{hm}}$ and lognormal standard deviations $\sigma$ obtained from analyses of the time and frequency domain measurements are listed in Table II. In general, it is seen that both $D_{\mathrm{hm}}$ and $\sigma$ increase with increasing concentration of bBSA. However, the values of $D_{\mathrm{hm}}$ obtained in the time domain do not differ significantly for bBSA concentrations of $2.5 \mathrm{nM}$ and $5 \mathrm{nM}$ (although the values of $\sigma$ do).

Figure 8 shows the values of $D_{\mathrm{hm}}$ extracted from fits of the measurements with $0 \mathrm{nM}$ and $10 \mathrm{nM}$ bBSA as function of time after sample injection. It is seen that in both the time and frequency domains, the sample without bBSA has a value of $D_{\mathrm{hm}}$ that increases slightly with time. In the presence of bBSA, the value of $D_{\mathrm{hm}}$ increases faster with time. It

TABLE II. Parameters obtained from least squares fitting to the measurement in the time and frequency domains for five different bBSA concentrations. The numbers in parentheses are the errors for the $95 \%$ confidence interval obtained from the least squares curve fits.

\begin{tabular}{llcllc}
\hline \hline & \multicolumn{2}{c}{ Time domain } & & \multicolumn{2}{c}{ Frequency domain } \\
\cline { 2 - 3 } \cline { 5 - 6 }$c_{\mathrm{bBSA}}[\mathrm{nm}]$ & $D_{\mathrm{hm}}[\mathrm{nm}]$ & $\sigma$ & & $D_{\mathrm{hm}}[\mathrm{nm}]$ & $\sigma$ \\
\hline 0 & $127.1(6)$ & $0.31(1)$ & & $129(2)$ & $0.34(2)$ \\
0 & $128.9(8)$ & $0.33(1)$ & & $130(2)$ & $0.34(2)$ \\
2.5 & $139.0(8)$ & $0.40(1)$ & & $137(2)$ & $0.40(3)$ \\
5 & $138.8(8)$ & $0.43(1)$ & & $147(3)$ & $0.47(3)$ \\
10 & $172.7(9)$ & $0.62(1)$ & & $190(4)$ & $0.65(3)$ \\
\hline \hline
\end{tabular}

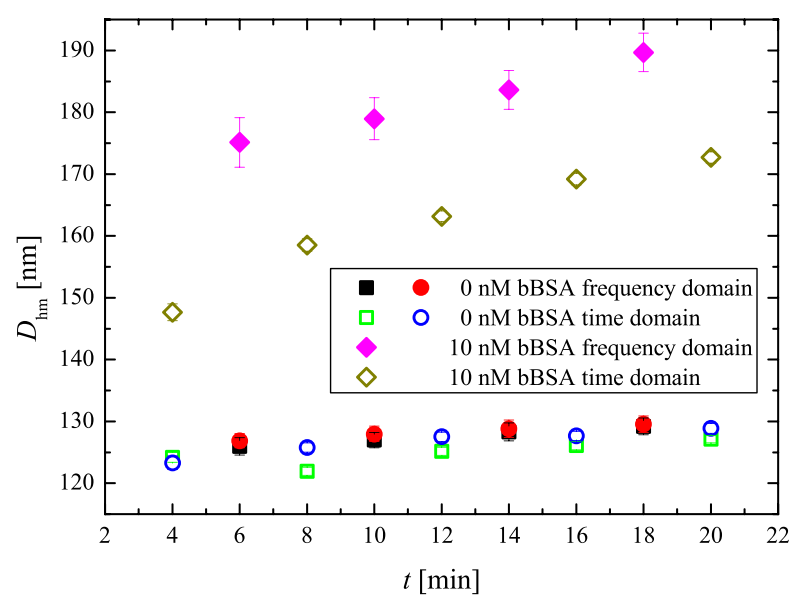

FIG. 8. Median hydrodynamic diameters obtained in the time and frequency domains vs. time after injection. Results are plotted for samples with $0 \mathrm{nM}$ and $10 \mathrm{nM}$ of bBSA.

is also seen that the values of $D_{\mathrm{hm}}$ obtained from frequency domain measurements are generally larger than those obtained from time domain measurements.

\section{DISCUSSION}

\section{A. Bead size}

For the beads with nominal sizes below $250 \mathrm{~nm}$, the hydrodynamic sizes found by the two methods agree within the experimental uncertainty. For the $250 \mathrm{~nm}$ beads, there are several possible explanations for the observed significant difference between the time and frequency domain measurements: The $250 \mathrm{~nm}$ bead suspension does not reach steadystate over the duration of the experiment and hence the signal continuously increases with time due to bead sedimentation. This means that the signal measured during a sweep in the frequency domain increases during the sweep such that the signal level is higher towards the end of the sweep. As the frequency is swept from high to low frequencies, this may skew the spectrum towards lower values, i.e., towards larger hydrodynamic diameters. In the time domain, each measurement is carried out over a much shorter time interval $(125 \mathrm{~ms})$ and is thus not affected by bead sedimentation. However, if not all beads can achieve equilibrium during a half-period, which is consistent with the observation for the $250 \mathrm{~nm}$ beads in Fig. 4, this will give a relatively larger weight to the small beads in the measurements and hence shift the result towards smaller bead dimensions.

The Brownian relaxation measurements of the $40 \mathrm{~nm}$ beads are almost on the limit of what can be resolved with the data acquisition card used for the time domain measurements, both in terms of signal level and time resolution. The signal relaxes in around $100 \mu$ s and due to the low pass filter, the first measurement is obtained after $17 \mu$ s and the time resolution of the data acquisition card is only $1.6 \mu \mathrm{s}$. This results in only 60 measurement points to define the relaxation signal. From the scattering of the points for the measurements on $40 \mathrm{~nm}$ beads, it is also seen that the signal change due to relaxation is only a few times the resolution of the data acquisition card. Note that a signal in these measurements of $1 \mu \mathrm{V}$ 
corresponds to an average magnetic field of $120 \mathrm{nT}$. Hence, at present, the time domain measurements are limited by the resolution and noise of the data acquisition card. Low-noise signal amplification can significantly improve this as the sensors have a very low intrinsic noise level. ${ }^{23}$ For example, the present sensors have a calculated thermal noise on the order of $1 \mathrm{nV}$ under the present measurement conditions. The time domain measurements can also be improved by magnetic and electrical shielding of the set-up and the inclusion of gradiometer-like sensor configurations. Such improvements are the focus of our ongoing studies.

\section{B. Bead clustering}

Both time and frequency domain measurements can be used to detect the presence of bBSA by measuring an increase in the hydrodynamic diameters. However, with the present setup and preliminary set of data, measurements in the frequency domain measurements are found to be more sensitive to bBSA. Again, this can likely be improved with lower noise electronics for the signal treatment and/or longer measuring times to avoid a skewing of the bead size distribution towards lower values.

It was found that for samples containing bBSA, the median hydrodynamic diameter increases with time, whereas without bBSA, it is nearly constant. This is because bead clusters sediment due to their larger size and they thereby increase the ratio between clustered and free beads near the sensor surface. This effect will potentially make the planar Hall effect bridge sensors more sensitive if longer time is waited before performing the measurement. Hence, an optimum condition can be defined from the stability of the free beads, the sedimentation time for the bead clusters, and the desired analysis time. Moreover, it can also be envisioned to use active bead manipulation, where the cluster sedimentation is accelerated using an externally applied magnetic field gradient.

\section{CONCLUSION}

We have shown that hydrodynamic sizes can be measured in a microfluidic channel with planar Hall effect bridge sensors in both the time and frequency domains without need for externally applied magnetic fields. We have also demonstrated that both methods can be used to detect clustering of streptavidin coated beads in the presence of biotinconjugated bovine serum albumin. In this study, the time domain measurements take only a sixth of the time of the measurements in the frequency domain and they provide results of similar quality. This time can potentially be further reduced by optimizing the electronics and measurement times on the order of seconds are therefore within reach. Such short measurement times will allow for quick diagnostic tests as well as for real-time monitoring of the binding kinetics of magnetic beads and biological samples.

\section{ACKNOWLEDGMENTS}

This work was supported by the Copenhagen Graduate School for Nanoscience and Nanotechnology (C:O:N:T) and the Knut and Alice Wallenberg (KAW) Foundation.
${ }^{1}$ I. Koh and L. Josephson, "Magnetic nanoparticle sensors," Sensors 9 , 8130 (2009).

${ }^{2} \mathrm{~S}$. X. Wang and G. Li, "Advances in giant magnetoresistance biosensors with magnetic nanoparticle tags: Review and outlook," IEEE Trans Magn. 44, 1687 (2008).

${ }^{3}$ N. Jaffrezic-Renault, C. Martelet, Y. Chevolot, and J.-P. Cloarec, "Biosensors and bio-bar code assays based on biofunctionalized magnetic microbeads," Sensors 7, 589 (2007).

${ }^{4}$ J. Göransson, T. Zardán Gómez De La Torre, M. Strömberg, C. Russell, P. Svedlindh, M. Strømme, and M. Nilsson, "Sensitive detection of bacterial DNA by magnetic nanoparticles," Anal. Chem. 82, 9138 (2010).

${ }^{5}$ J. Schotter, P. Kamp, A. Becker, A. Pühler, G. Reiss, and H. Brückl, "Comparison of a prototype magnetoresistive biosensor to standard fluorescent DNA detection," Biosens. Bioelectron. 19, 1149 (2004).

${ }^{6}$ R. S. Gaster, D. A. Hall, C. H. Nielsen, S. J. Osterfeld, H. Yu, K. E. Mach, R. J. Wilson, B. Murmann, J. C. Liao, S. S. Gambhir, and S. X. Wang, "Matrix-insensitive protein assays push the limits of biosensors in medicine," Nat. Med. 15, 1327 (2009).

${ }^{7}$ J. Connolly and T. G. St Pierre, "Proposed biosensors based on timedependent properties of magnetic fluids," J. Magn. Magn. Mater. 225, 156 (2001).

${ }^{8}$ A. P. Astalan, F. Ahrentorp, C. Johansson, K. Larsson, and A. Krozer, "Biomolecular reactions studied using changes in Brownian rotation dynamics of magnetic particles," Biosens. Bioelectron. 19, 945 (2004).

${ }^{9}$ F. Ludwig, S. Mäuselein, E. Heim, and M. Schilling, "Magnetorelaxometry of magnetic nanoparticles in magnetically unshielded environment utilizing a differential fluxgate arrangement," Rev. Sci. Instrum. 76, 106102 (2005).

${ }^{10}$ H. Grossman, W. Myers, V. Vreeland, R. Bruehl, M. Alper, C. Bertozzi, and J. Clarke, "Detection of bacteria in suspension by using a superconducting quantum interference device," Proc. Natl. Acad. Sci. U.S.A. 101, 129 (2004).

${ }^{11}$ B. T. Dalslet, C. D. Damsgaard, M. Donolato, M. Strømme, M. Strömberg, P. Svedlindh, and M. F. Hansen, "Bead magnetorelaxometry with an on-chip magnetoresistive sensor," Lab Chip 11, 296 (2011).

${ }^{12}$ F. W. Østerberg, B. T. Dalslet, D. Snakenborg, C. Johansson, and M. F. Hansen, "Chip-based measurements of Brownian relaxation of magnetic beads using a planar hall effect magnetic field sensor," AIP Conf. Proc. 1311, 176-183 (2010).

${ }^{13}$ R. Kötitz, P. Fannin, and L. Trahms, "Time domain study of Brownian and Néel relaxation in ferrofluids," J. Magn. Magn. Mater. 149, 42 (1995).

${ }^{14}$ D. Eberbeck, C. Bergemann, F. Wiekhorst, U. Steinhoff, and L. Trahms, "Quantification of specific bindings of biomolecules by magnetorelaxometry," NanoBiotechnology 6, 4 (2008).

${ }^{15}$ F. Öisjöen, J. F. Schneiderman, A. P. Astalan, A. Kalabukhov, C. Johansson, and D. Winkler, "A new approach for bioassays based on frequency- and time-domain measurements of magnetic nanoparticles," Biosens. Bioelectron. 25, 1008 (2010).

${ }^{16}$ F. W. Østerberg, G. Rizzi, T. Zardán Gómez de la Torre, M. Strömberg, M. Strømme, P. Svedlindh, and M. F. Hansen, "Measurements of Brownian relaxation of magnetic nanobeads using planar hall effect bridge sensors," Biosens. Bioelectron. 40, 147 (2013).

${ }^{17}$ F. W. Østerberg, G. Rizzi, and M. F. Hansen, "On-chip measurements of Brownian relaxation of magnetic beads with diameters from $10 \mathrm{~nm}$ to 250 nm,” J. Appl. Phys. 113, 154507 (2013).

${ }^{18}$ L. Neél, "Théorie du traînage magnétique des ferromagnétiques en grains fins avec applications aux terres cuites," Ann. Géophys. 5, 99 (1949).

${ }^{19}$ W. Brown, "Thermal fluctuations of a single-domain particle," Phys. Rev. 130, 1677 (1963).

${ }^{20}$ P. Debye, Polar Molecules (Chemical Catalogue Co., 1929).

${ }^{21}$ A. D. Henriksen, B. T. Dalslet, D. H. Skieller, K. H. Lee, F. Okkels, and M. F. Hansen, "Planar Hall effect bridge magnetic field sensors," Appl. Phys. Lett. 97, 013507 (2010).

${ }^{22}$ T. B. G. Hansen, C. D. Damsgaard, B. T. Dalslet, and M. F. Hansen, "Theoretical study of in-plane response of magnetic field sensor to magnetic beads magnetized by the sensor self-field," J. Appl. Phys. 107, 124511 (2010).

${ }^{23}$ A. Persson, R. S. Bejhed, H. Nguyen, K. Gunnarsson, B. T. Dalslet, F. W. Østerberg, M. F. Hansen, and P. Svedlindh, "Low-frequency noise in planar Hall effect bridge sensors," Sens. Actuators, A 171, 212 (2011). 